\title{
A Case Report of Severe Corneal Toxicity following $0.5 \%$ Topical Moxifloxacin Use
}

\author{
A.P. Vignesh Renuka Srinivasan Swathi Karanth \\ Department of Ophthalmology, Jawaharlal Institute of Postgraduate Medical Education \\ and Research, Pondicherry, India
}

\section{Key Words}

Topical moxifloxacin · Fluoroquinolones · Keratitis

\begin{abstract}
Moxifloxacin is a widely used topical antibiotic in various bacterial infections of the eye. Its safety and efficacy have been proved by many studies. We report a case of a rare adverse effect following its use. A 10-year-old female who had presented with acute bacterial conjunctivitis in both eyes with no corneal involvement was started on preservative-free $0.5 \%$ topical moxifloxacin four times a day. The child developed a severe form of corneal toxicity in both eyes with circumcorneal congestion and corneal edema following its use. The child's visual acuity had dropped from $20 / 20$ to $20 / 400$ in both the eyes. Topical moxifloxacin was discontinued, following which the cornea cleared dramatically and the visual acuity became normal. This case indicates that though rare, topical moxifloxacin can cause severe keratitis and that more studies need to be conducted to evaluate its safety.

(c) 2015 S. Karger AG, Basel
\end{abstract}

\section{Introduction}

Moxifloxacin, a fourth-generation fluoroquinolone is a broad-spectrum antibiotic effective in various ocular bacterial infections like conjunctivitis, corneal ulcers and endophthalmitis [1]. Various studies have shown it to be relatively safe and have reported only very mild side effects [2]. We report a case in which the patient developed a severe form of keratitis following its use, and on discontinuing the drug the condition resolved. 
Vignesh et al.: A Case Report of Severe Corneal Toxicity following 0.5\% Topical Moxifloxacin Use

\section{Case Report}

A 10-year-old female from India presented to our outpatient department with redness and discharge in both eyes. Her visual acuity was $20 / 20$ in both the eyes. On examination, she had conjunctival congestion in both eyes with mucopurulent discharge. The cornea was clear and the rest of the ocular examination was normal. A conjunctival swab was taken, and it showed Gram-positive cocci on Gram staining. A diagnosis of acute mucopurulent conjunctivitis of bacterial origin was made. She was started on $0.5 \%$ preservative-free moxifloxacin four times a day and kept on follow-up. Three days later, she came back with severe pain, redness, photophobia, watering and a drop in visual acuity in both the eyes. On examination, her visual acuity was $20 / 400$, and she had circumcorneal congestion with corneal edema (fig. 1). There was a small epithelial defect with superficial punctate keratitis in both the eyes. The corneal sensation was normal, and the intraocular pressure was $10 \mathrm{~mm} \mathrm{Hg}$ in both the eyes. Her tear function tests were normal. The patient was not on any other topical medications. Swabs from the conjunctiva were sent for bacterial and fungal culture sensitivity. Polymerase chain reaction analysis was also done to look for a viral etiology. All the laboratory tests turned out to be negative. A diagnosis of possible drug toxicity was made and topical moxifloxacin was discontinued. Her cornea showed signs of clearing, and her vision returned to normal in the next week (fig. 1).

\section{Discussion}

Topical moxifloxacin is emerging as the choice of antibiotic in bacterial infections of the eye because of its broad spectrum of action and clinical efficacy, and it is preservative free. Studies have demonstrated that it is relatively safe in clinical conditions. Some of the side effects reported with its use are conjunctivitis, ocular hyperemia, dry eye, irritation and watering, all of which were mild and transient [3]. Walter and Tyler [4] reported 2 cases of worsening of corneal ulcer after moxifloxacin treatment. In our case, the patient developed a severe form of corneal toxicity with corneal edema and superficial punctate keratitis following topical moxifloxacin use. Unlike the earlier reports, the cornea was not involved in the disease process before starting the medication, and the condition was quite severe with the patient experiencing a drop in vision. The patient recovered dramatically after stopping the medication. This is a severe and rare case of corneal toxicity following topical moxifloxacin use. Experimental studies have suggested the mechanism of toxicity of moxifloxacin to be due to corneal epithelial degradation, inhibition of collagen IV synthesis, damage to Descemet's membrane [5] and breakdown of tight junctions of corneal epithelium [6].

In summary, this is a rare case of corneal toxicity which occurred following the use of topical moxifloxacin, and the effect reversed dramatically following its discontinuation. Though there are reports of toxicity of moxifloxacin, they all document either mild cases or cases where the cornea was already affected, which was due to the drug interfering with the healing process. In our case, the cornea was uninvolved prior to the drug use and it was the most probable cause of the toxicity. This case indicates that further studies are needed to study the safety of moxifloxacin because, though rare, it can lead to a severe form of corneal toxicity with loss of vision. 
Case Reports in

Ophthalmology

\section{Disclosure Statement}

None of the authors has any financial/conflicting interests to disclose.

\section{References}

1 Wong RLM, Gangwani RA, Yu LWH, Lai JSM: New treatments for bacterial keratitis. J Ophthalmol 2012;2012:831502.

-2 Miller D: Review of moxifloxacin hydrochloride ophthalmic solution in the treatment of bacterial eye infections. Clin Ophthalmol 2008;2:77-91.

-3 Hariprasad SM, Blinder KJ, Shah GK, Apte RS, Rosenblatt B, Holekamp NM, et al: Penetration pharmacokinetics of topically administered $0.5 \%$ moxifloxacin ophthalmic solution in human aqueous and vitreous. Arch Ophthalmol 2005;123:39-44.

$\checkmark 4$ Walter K, Tyler ME: Severe corneal toxicity after topical fluoroquinolone therapy: report of two cases. Cornea 2006;25:855-857.

-5 Stern ME, Gao J, Beuerman RW, Farley W, Zhuo L, McDonnell PJ, et al: Effects of fourth-generation fluoroquinolones on the ocular surface, epithelium, and wound healing. Cornea 2006;25(suppl 2):S12-S24.

-6 Ly LT, Cavanagh HD, Petroll WM: Confocal assessment of the effects of fourth-generation fluoroquinolones on the cornea. Eye Contact Lens 2006;32:161-165.

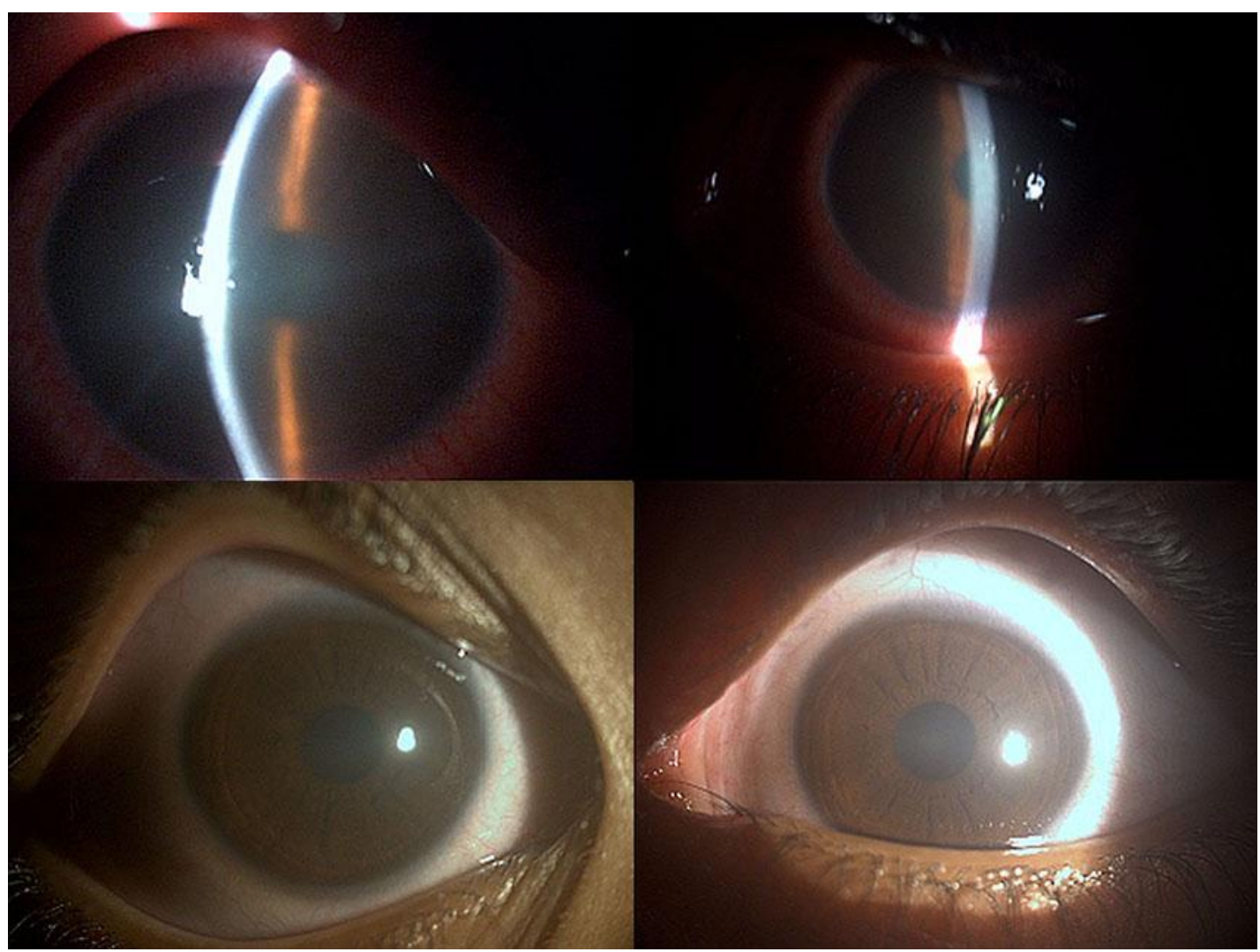

Fig. 1. Severe keratitis following moxifloxacin use which resolved after discontinuing the drug. 